\title{
Analysis and dynamically consistent nonstandard discretization for a rabies model in humans and dogs
}

\author{
M. Chapwanya, J. M. -S. Lubuma, and Y. A. Terefe \\ Department of Mathematics and Applied Mathematics, \\ University of Pretoria
}

\begin{abstract}
Rabies is a fatal disease in dogs as well as in humans. A possible model to represent rabies transmission dynamics in human and dog populations is presented. The next generation matrix operator is used to determine the threshold parameter $\mathcal{R}_{0}$, that is the average number of new infective individuals produced by one infective individual introduced into a completely susceptible population. If $\mathcal{R}_{0}<1$, the disease-free equilibrium is globally asymptotically stable, while it is unstable and there exists a locally asymptotically stable endemic equilibrium when $\mathcal{R}_{0}>1$. A nonstandard finite difference scheme that replicates the dynamics of the continuous model is proposed. Numerical tests to support the theoretical analysis are provided.
\end{abstract}

keywords: Rabies, Rabies model, disease-free equilibrium, endemic equilibrium, nonstandard finite difference scheme, dynamics preserving scheme.

Mathematics Subject Classification: 37N25, 37N30, 65C20, 92B05. 


\section{Introduction}

Rabies, also known as hydrophobia of human, is an acute disease that progresses to coma or death after the first symptom appearers by attacking the central nervous system of the victim. It is a viral disease that mainly affects carnivores and bats, although it can affect any mammals. Rabies transmits from animals to animals or animals to humans through bites or scratches [10]. The most common wild animals to transmit rabies are raccoons, skunks, bats, foxes and coyotes while domestic animals such as cats, cattle and dogs can also transmit rabies $[9]$.

A major observation during infection is a change in personality of the rabid animal such as approach humans without fear or hesitation. Many rabid animals are incapable of swallowing their saliva. The incubation period, the time between exposure to the rabies virus and the development of sign varies from one week to one year.

The primary route of humans infection with the rabies is through a bite from a rabid dog. Rabies transmission is also possible when a claw scratch or mucosal surface (e.g. mouse, nose,

eye) is contaminated with the saliva from a rabid dog. Rabies is responsible for the death of 50,000 to 60,000 people annually although effective vaccines for pre and post-exposed individuals are available. More than 99 percent of these death occur in the developing countries where the disease is endemic in domestic dog population. There is no treatment after clinical symptoms are observed $[3,10]$.

Domestic dogs in most African countries are the major source of rabies disease for humans. Understanding the dynamics of rabies between humans and domestic dogs would therefore important to design a more effective control strategy that could reduce human deaths. In the controlling strategies of the disease, mathematical models play significant roles.

A model to study the spread of rabies among foxes is given in [17]. Here, we develop a model to study the rabies transmission between human and dog populations. In each population of our study, we have susceptible (S), exposed (E), and infectious (I) compartments. In the human population, we have additional compartment denoted by (V) containing indi- 
viduals removed by vaccination.

The proposed model, like most mathematical models in applied sciences, cannot be solved by analytical techniques. Here, we design a nonstandard finite difference (NSFD) scheme to approximate the solution of the continuous model. The scheme is constructed based on two Mickens' rules [15] and as singled out in [2]. To the best of our knowledge, this is the first time to present the rabies model between human and dog populations with its dynamically consistent numerical approximation.

This article is organized as follows. The model is formulated in Section 2. Equilibrium points and their stability analysis are given in Section 3. In Section 4, a dynamically consistent NSFD scheme is designed and its numerical simulations are illustrated in Section 5. Concluding remarks on our findings and possible future research directions are indicated in the last Section.

\section{Model formulation}

To formulate the model, we make the following assumptions:

1. there is no transmission of the disease from rabid humans to susceptible humans;

2. there is no contacts between rabid humans and susceptible dogs;

3. the contacts among susceptible and exposed humans, and among susceptible and exposed dogs don't give rise to infection;

4. there is no vaccination for susceptible dogs before and after exposed to the disease.

Based on these assumptions, the dynamics of the disease between humans and dogs is given in Fig 1. 


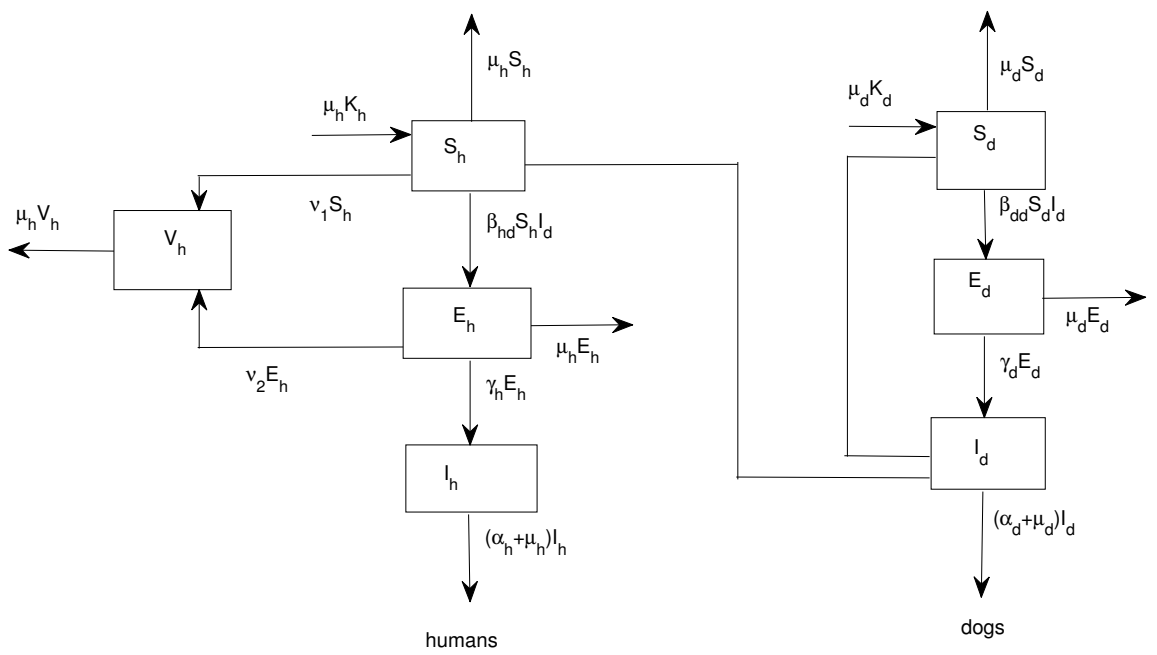

Figure 1: Flow chart

Using system of differential equations, the flow chart given in Fig 1 reads as follows:

$$
\begin{aligned}
S_{h}^{\prime} & =\mu_{h} K_{h}-\beta_{h d} S_{h} I_{d}-\mu_{h} S_{h}-\nu_{1} S_{h} \\
E_{h}^{\prime} & =\beta_{h d} S_{h} I_{d}-\left(\mu_{h}+\gamma_{h}+\nu_{2}\right) E_{h} \\
I_{h}^{\prime} & =\gamma_{h} E_{h}-\left(\alpha_{h}+\mu_{h}\right) I_{h} \\
V_{h}^{\prime} & =\nu_{1} S_{h}+\nu_{2} E_{h}-\mu_{h} V_{h} \\
S_{d}^{\prime} & =\mu_{d} K_{d}-\beta_{d d} S_{d} I_{d}-\mu_{d} S_{d} \\
E_{d}^{\prime} & =\beta_{d d} S_{d} I_{d}-\left(\mu_{d}+\gamma_{d}\right) E_{d} \\
I_{d}^{\prime} & =\gamma_{d} E_{d}-\left(\alpha_{d}+\mu_{d}\right) I_{d},
\end{aligned}
$$


where all parameters are nonnegative and the description of these parameters are given in Table 1. Moreover, $N_{h}=S_{h}+E_{h}+I_{h}+V_{h}$ and $N_{d}=S_{d}+E_{d}+I_{d}$. Furthermore $N_{h}$ and $N_{d}$ satisfy the equations

$$
N_{h}^{\prime}=\mu_{h} K_{h}-\mu_{h} N_{h}-\alpha_{h} I_{h}
$$

and

$$
N_{d}^{\prime}=\mu_{d} K_{d}-\mu_{d} N_{d}-\alpha_{d} I_{d}
$$

respectively. Nonnegative solutions $N_{h}$ for (2.8) and $N_{d}$ for (2.9) satisfy the conservation laws

$$
\mu_{h} K_{h}-\left(\mu_{h}+\alpha_{h}\right) N_{h} \leq N_{h}^{\prime} \leq \mu_{h}\left(K_{h}-N_{h}\right)
$$

and

$$
\mu_{d} K_{d}-\left(\mu_{d}+\alpha_{d}\right) N_{d} \leq N_{d}^{\prime} \leq \mu_{d}\left(K_{d}-N_{d}\right)
$$

respectively [16]. By applying Gronwall inequality for differential equations [18], from above and below of (2.10) and (2.11), we get for $t \geq 0$,

$$
\frac{\mu_{h} K_{h}}{\mu_{h}+\alpha_{h}}-\left(\frac{\mu_{h} K_{h}}{\mu_{h}+\alpha_{h}}-N_{h}^{0}\right) e^{-\left(\mu_{h}+\alpha_{h}\right) t} \leq N_{h}(t) \leq K_{h}-\left(K_{h}-N_{h}^{0}\right) e^{-\mu_{h} t}
$$

and

$$
\frac{\mu_{d} K_{d}}{\mu_{d}+\alpha_{d}}-\left(\frac{\mu_{d} K_{d}}{\mu_{d}+\alpha_{d}}-N_{d}^{0}\right) e^{-\left(\mu_{d}+\alpha_{d}\right) t} \leq N_{d}(t) \leq K_{d}-\left(K_{d}-N_{d}^{0}\right) e^{-\mu_{d} t},
$$

respectively. Thus for $0 \leq N_{h}^{0} \leq K_{h}$ and $0 \leq N_{d}^{0} \leq K_{d}$, we get respectively,

$$
0 \leq N_{h}(t) \leq K_{h} \quad \text { and } \quad 0 \leq N_{d}(t) \leq K_{d}
$$

Theorem 2.1 The epidemiological model (2.1) - (2.7) defines a dynamical system on the biologically feasible region

$$
\begin{aligned}
\Omega= & \left\{\left(S_{h}, E_{h}, I_{h}, V_{h}, S_{d}, E_{d}, I_{d}\right) \in \mathbb{R}_{+}^{7}: 0 \leq S_{h}+E_{h}+I_{h}+V_{h}=N_{h} \leq K_{h},\right. \\
& \left.0 \leq S_{d}+E_{d}+I_{d}=N_{d} \leq K_{d}\right\} .
\end{aligned}
$$




\begin{tabular}{|c|c|c|c|}
\hline $\begin{array}{c}\text { Parameters } \\
\text { and variables }\end{array}$ & Description & $\begin{array}{c}\text { Parameters } \\
\text { and variables }\end{array}$ & Description \\
\hline$K_{h}$ & human carrying capacity & $S_{h}$ & susceptible human \\
$E_{h}$ & exposed human & $I_{h}$ & Infectious human \\
$V_{h}$ & vaccinated human pre-exposed and post-exposed & $\mu_{h}$ & birth or natural death human \\
$\beta_{h d}$ & contact rate between $S_{h}$ and $I_{d}$ & $\nu_{1}$ & rate of vaccination for $S_{h}$ \\
$\gamma_{h}$ & rate of transfer from $E_{h}$ to $I_{h}$ & $\nu_{2}$ & rate of vaccination for $E_{h}$ \\
$\alpha_{h}$ & death rate of human due to rabies & $K_{d}$ & dog carrying capacity \\
$S_{d}$ & susceptible dog & $E_{d}$ & exposed dog \\
$I_{d}$ & infectious dog & $\mu_{d}$ & birth or natural death rate of dogs \\
$\beta_{d d}$ & contact rate between $S_{d}$ and $I_{d}$ & $\gamma_{d}$ & rate of transfer from $E_{d}$ to $I_{d}$ \\
$\alpha_{d}$ & death rate of dogs induced by rabies & & \\
\hline
\end{tabular}

Table 1: Description of variables and parameters.

Proof: The theorem can be proved by showing that $\Omega$ is positively invariant. Thus for any initial data in $\Omega$, no trajectory will leave $\Omega$ by crossing through one of its faces (see [5]).

\section{$3 \quad$ Equilibrium points and their stability analysis}

To determine the equilibrium points of $(2.1)-(2.7)$, we set

$$
\begin{aligned}
\mu_{h} K_{h}-\beta_{h d} S_{h} I_{d}-\mu_{h} S_{h}-\nu_{1} S_{h} & =0 \\
\beta_{h d} S_{h} I_{d}-\left(\mu_{h}+\gamma_{h}+\nu_{2}\right) E_{h} & =0 \\
\gamma_{h} E_{h}-\left(\alpha_{h}+\mu_{h}\right) I_{h} & =0 \\
\nu_{1} S_{h}+\nu_{2} E_{h}-\mu_{h} V_{h} & =0 \\
\mu_{d} K_{d}-\beta_{d d} S_{d} I_{d}-\mu_{d} S_{d} & =0 \\
\beta_{d d} S_{d} I_{d}-\left(\mu_{d}+\gamma_{d}\right) E_{d} & =0 \\
\gamma_{d} E_{d}-\left(\alpha_{d}+\mu_{d}\right) I_{d} & =0 .
\end{aligned}
$$


From (3.7), we obtain

$$
E_{d}=\frac{\left(\alpha_{d}+\mu_{d}\right) I_{d}}{\gamma_{d}}
$$

Inserting Equation (3.8) into Equation (3.6), we get

$$
\beta_{d d} S_{d} I_{d}-\frac{\left(\gamma_{d}+\mu_{d}\right)\left(\alpha_{d}+\mu_{d}\right) I_{d}}{\gamma_{d}}=0
$$

This gives $I_{d}=0$ or $\beta_{d d} S_{d}-\frac{\left(\gamma_{d}+\mu_{d}\right)\left(\alpha_{d}+\mu_{d}\right)}{\gamma_{d}}=0$.

If $I_{d}=0$, then

$$
E_{0} \equiv\left(S_{h}, E_{h}, I_{h}, V_{h}, S_{d}, E_{d}, I_{d}\right)=\left(\frac{\mu_{h} K_{h}}{\mu_{h}+\nu_{1}}, 0,0, \frac{\nu_{1} K_{h}}{\mu_{h}+\nu_{1}}, K_{d}, 0,0\right)
$$

is the disease-free equilibrium obtained by using appropriate substitutions in the subsequent equations.

To find the basic reproduction number which is very important in the qualitative analysis of the model, we use the method of next generation matrix discussed in $[6,20]$. For this, we consider the vector function

$$
F=\left(\begin{array}{c}
\beta_{h d} S_{h} I_{d} \\
0 \\
\beta_{d d} S_{d} I_{d} \\
0
\end{array}\right)
$$

which is the rate at which disease compartments increase in size, but the vector function

$$
V=\left(\begin{array}{c}
\left(\gamma_{h}+\mu_{h}+\nu_{2}\right) E_{h} \\
-\gamma_{h} E_{h}+\left(\alpha_{h}+\mu_{h}\right) I_{h} \\
\left(\mu_{d}+\gamma_{d}\right) E_{d} \\
-\gamma_{d} E_{d}+\left(\alpha_{d}+\mu_{d}\right) I_{d}
\end{array}\right)
$$


is the rate at which the disease compartments decrease due to the disease. By using Diekmann et al [6] and van Driessche et al [20], the next generation matrix is given by

$$
K=J_{F}\left(J_{V}\right)^{-1},
$$

where

$$
J_{F}=\left(\begin{array}{cccc}
0 & 0 & 0 & \frac{\beta_{h d} \mu_{h} K_{h}}{\mu_{h}+\nu_{1}} \\
0 & 0 & 0 & 0 \\
0 & 0 & 0 & \beta_{d d} K_{d} \\
0 & 0 & 0 & 0
\end{array}\right)
$$

is the Jacobian matrix of $F$ at $E_{0}$ and

$$
J_{V}=\left(\begin{array}{cccc}
\gamma_{h}+\mu_{h}+\nu_{2} & 0 & 0 & 0 \\
-\gamma_{h} & \alpha_{h}+\mu_{h} & 0 & 0 \\
0 & 0 & \gamma_{d}+\mu_{d} & 0 \\
0 & 0 & -\gamma_{d} & \alpha_{d}+\mu_{d}
\end{array}\right)
$$

is the Jacobian of $V$ at $E_{0}$. The basic reproduction number denoted by $\mathcal{R}_{0}$ is defined as the expected number of secondary cases produced in a completely susceptible population by a typical infected individual during its entire period of infectious [4]. Numerically, it is defined as the spectral radius of $K$. That is $\mathcal{R}_{0}=\rho(K)$ and explicitly,

$$
\mathcal{R}_{0}=\frac{\gamma_{d} \beta_{d d} K_{d}}{\left(\gamma_{d}+\mu_{d}\right)\left(\alpha_{d}+\mu_{d}\right)} .
$$

Remark 3.1 In Equation (3.10), we can see that $\mathcal{R}_{0}$ is completely defined in terms of the parameters of the dog population. In the eradication strategy of the disease, the action to decrease the value of $\mathcal{R}_{0}$ is advised to considered with respect to these parameters.

If $I_{d}>0, \quad$ from (3.9)

$$
S_{d}=\frac{\left(\gamma_{d}+\mu_{d}\right)\left(\alpha_{d}+\mu_{d}\right)}{\gamma_{d} \beta_{d d}} \quad \text { or } \quad S_{d}=\frac{K_{d}}{\mathcal{R}_{0}}
$$


Then by back substitution,

$$
E_{\infty}=\left(S_{h}^{*}, E_{h}^{*}, I_{h}^{*}, V_{h}^{*}, S_{d}^{*}, E_{d}^{*}, I_{d}^{*}\right)
$$

is the endemic equilibrium, where

$$
\begin{aligned}
& S_{h}^{*}=\frac{\mu_{h} \beta_{d d} K_{h}}{\mu_{d} \beta_{h d}\left(\mathcal{R}_{0}-1\right)+\left(\mu_{h}+\nu_{1}\right) \beta_{d d}}, \\
& E_{h}^{*}=\frac{\mu_{d} \mu_{h} \beta_{h d} K_{h}\left(\mathcal{R}_{0}-1\right)}{\left(\mu_{h}+\gamma_{h}+\nu_{2}\right)\left[\mu_{d} \beta_{h d}\left(\mathcal{R}_{0}-1\right)+\left(\mu_{h}+\nu_{1}\right) \beta_{d d}\right]}, \\
& I_{h}^{*}=\frac{\mu_{d} \mu_{h} \gamma_{h} \beta_{h d} K_{h}\left(\mathcal{R}_{0}-1\right)}{\left(\alpha_{h}+\mu_{h}\right)\left(\mu_{h}+\gamma_{h}+\nu_{2}\right)\left[\mu_{d} \beta_{h d}\left(\mathcal{R}_{0}-1\right)+\left(\mu_{h}+\nu_{1}\right) \beta_{d d}\right]}, \\
& V_{h}^{*}=\frac{K_{h}}{\mu_{d} \beta_{h d}\left(\mathcal{R}_{0}-1\right)+\left(\mu_{h}+\nu_{1}\right) \beta_{d d}}\left(\nu_{1} \beta_{d d}+\frac{\nu_{2} \mu_{d} \beta_{h d}\left(\mathcal{R}_{0}-1\right)}{\mu_{h}+\gamma_{h}+\nu_{2}}\right) \\
& S_{d}^{*}=\frac{K_{d}}{\mathcal{R}_{0}}, \quad E_{d}^{*}=\frac{\mu_{d}\left(\alpha_{d}+\mu_{d}\right)\left(\mathcal{R}_{0}-1\right)}{\gamma_{d} \beta_{d d}}, \quad \text { and } \quad I_{d}^{*}=\frac{\mu_{d}}{\beta_{d d}}\left(\mathcal{R}_{0}-1\right) .
\end{aligned}
$$

For $\mathcal{R}_{0}>1$, we obtain unique endemic equilibrium.

Theorem 3.2 The disease-free equilibrium point $E_{0}$ is globally asymptotically stable for $\mathcal{R}_{0}<$ 1 and unstable for $\mathcal{R}_{0}>1$.

Proof: To show that $E_{0}$ is globally asymptotically stable, we use the LaSalle Invariance Principle [12].

Let $E(t)=\left(S_{h}(t), E_{h}(t), I_{h}(t), V_{d}(t), S_{d}(t), E_{d}(t), I_{d}(t)\right) \in \mathbb{R}_{+}^{7}$ for $t>0$, then we consider a function defined by

$$
\begin{aligned}
U(E) & =\gamma_{d} E_{d}+\left(\mu_{d}+\gamma_{d}\right) I_{d} \\
\dot{U}(E) & =\gamma_{d} E_{d}^{\prime}+\left(\mu_{d}+\gamma_{d}\right) I_{d}^{\prime} \\
& =\gamma_{d} \beta_{d d} S_{d} I_{d}-\left(\mu_{d}+\gamma_{d}\right)\left(\alpha_{d}+\mu_{d}\right) I_{d} \\
& \leq\left(\gamma_{d} \beta_{d d} K_{d}-\left(\mu_{d}+\gamma_{d}\right)\left(\alpha_{d}+\mu_{d}\right)\right) I_{d} \\
& \leq\left(\mu_{d}+\gamma_{d}\right)\left(\alpha_{d}+\mu_{d}\right)\left(\mathcal{R}_{0}-1\right) I_{d} \\
& <0
\end{aligned}
$$


for $\mathcal{R}_{0}<1$ and $E \neq E_{0}$. Hence, $U$ is a positive definite Lyapunov function and $E_{0}$ is locally asymptotically stable for $\mathcal{R}_{0}<1$ while unstable for $\mathcal{R}_{0}>1$. By using the procedure mention in $[12,19]$, it is not difficult to show that the set $A=\left\{E_{0}\right\}$ is the maximum invariant set in $\mathcal{M}=\{E: \dot{U}=0\}$ for $\mathcal{R}_{0}<1$. This completes the proof.

Remark 3.3 By using the Hartman-Grobman theorem [18], we can prove that $E_{0}$ is hyperbolic, locally asymptotically stable for $\mathcal{R}_{0}<1$ and unstable for $\mathcal{R}_{0}>1$.

For $I_{d}>0$ and $\mathcal{R}_{0}>1$, the stability property of $E_{\infty}$ is given in the next theorem.

Theorem 3.4 For $\mathcal{R}_{0}>1$, the endemic equilibrium point $E_{\infty}$ is locally asymptotically stable.

Proof: To prove this theorem, we use Hartman-Grobman theorem [18]. The Jacobian matrix of the right-side of $(2.1)-(2.7)$ at $E_{\infty}$ is

\begin{tabular}{|c|c|c|c|c|c|c|}
\hline$-\left(\beta_{h d} I_{d}^{*}+\mu_{h}+\nu_{1}\right)$ & 0 & 0 & 0 & 0 & 0 & $-\beta_{h d} S_{h}^{*}$ \\
\hline$\beta_{h d} I_{d}^{*}$ & $-\left(\mu_{h}+\gamma_{h}+\nu_{2}\right)$ & 0 & 0 & 0 & 0 & $\beta_{h d} S_{h}^{*}$ \\
\hline 0 & $\gamma_{h}$ & $-\left(\alpha_{h}+\mu_{h}\right)$ & 0 & 0 & 0 & 0 \\
\hline$\nu_{1}$ & $\nu_{2}$ & 0 & $-\mu_{h}$ & 0 & 0 & 0 \\
\hline 0 & 0 & 0 & 0 & $-\left(\beta_{d d} I_{d}^{*}+\mu_{d}\right)$ & 0 & $-\beta_{d d} S_{d}^{*}$ \\
\hline 0 & 0 & 0 & 0 & $\beta_{d d} I_{d}^{*}$ & $-\left(\mu_{d}+\gamma_{d}\right)$ & $\beta_{d d} S_{d}^{*}$ \\
\hline 0 & 0 & 0 & 0 & 0 & $\gamma_{d}$ & $-\left(\alpha_{d}+\mu_{d}\right.$ \\
\hline
\end{tabular}

Clearly, the trace of this matrix is negative. We need to show that all eigenvalues of $J\left(E_{\infty}\right)$ have negative real parts. For this, let $r$ be an eigenvalue of $J\left(E_{\infty}\right)$ and the characteristic equation is

$$
\operatorname{det}\left(r I-J\left(E_{\infty}\right)\right)=0,
$$


which leads into the equation

$$
\begin{gathered}
{\left[r+\left(\beta_{h d} I_{d}^{*}+\mu_{h}+\nu_{1}\right)\right]\left(r+\left(\mu_{h}+\gamma_{h}+\nu_{2}\right)\right)\left[r+\mu_{h}\right]\left\{\left[r+\left(\beta_{d d} I_{d}^{*}+\mu_{d}\right)\right]\left(r+\left(\mu_{d}+\gamma_{d}\right)\right)\left[r+\left(\alpha_{d}+\mu_{d}\right)\right]\right.} \\
\left.-\left(r+\mu_{d}\right) \gamma_{d} \beta_{d d} S_{d}^{*}\right\}=0 .
\end{gathered}
$$

All real parts of $r$ in Equation (3.11) are negative if and only if all $r$ in

$$
\left[r+\left(\beta_{d d} I_{d}^{*}+\mu_{d}\right)\right]\left(r+\left(\mu_{d}+\gamma_{d}\right)\right)\left[r+\left(\alpha_{d}+\mu_{d}\right)\right]-\left(r+\mu_{d}\right) \gamma_{d} \beta_{d d} S_{d}^{*}=0
$$

have negative real parts. By using values of $I_{d}^{*}$ and $S_{d}^{*}$ with $\mathcal{R}_{0}$ in (3.10), Equation (3.12) can be written as

$$
r^{3}+a_{2} r^{2}+a_{1} r+a_{0}=0
$$

where,

$$
\begin{aligned}
& a_{2}=\alpha_{d}+\mu_{d}\left(\mathcal{R}_{0}+2\right)+\gamma_{d} \\
& a_{1}=\mu_{d} \mathcal{R}_{0}\left(\alpha_{d}+2 \mu_{d}+\gamma_{d}\right) \\
& a_{0}=\mu_{d}\left(\mu_{d}+\gamma_{d}\right)\left(\alpha_{d}+\mu_{d}\right)\left(\mathcal{R}_{0}-1\right)
\end{aligned}
$$

Clearly $a_{2}>0$, because the parameters in the expression are positive. It is also possible to show that $a_{2} a_{1}>a_{0}>0$ for $\mathcal{R}_{0}>1$. Then by Routh-Hurwitz condition [8], all real parts of $r$ in (3.13) are negative. Hence, the endemic equilibrium point, $E_{\infty}$ is locally asymptotically stable for $\mathcal{R}_{0}>1$.

\section{Nonstandard finite difference scheme}

In this section, we want to design a nonstandard finite difference scheme for $(2.1)-(2.7)$ that preserves all properties we discussed in the continuous model. For this numerical approximation, we replace the continuous time $t \in[0, \infty)$ with $t_{n}=n \Delta t, n=0,1,2, \ldots$, where $\Delta t$ is the time step-size. Thus, we are interested in finding approximate solutions 
$S_{h}^{n}, E_{h}^{n}, I_{h}^{n}, V_{h}^{n}, S_{d}^{n}, E_{d}^{n}, I_{d}^{n}$ of $S_{h}, E_{h}, I_{h}, V_{h}, S_{d}, E_{d}, I_{d}$ at time $t=t_{n}$, respectively. Having these in mind, we propose the following NSFD scheme:

$$
\begin{aligned}
\frac{S_{h}^{n+1}-S_{h}^{n}}{\phi(\Delta t)} & =\mu_{h} K_{h}-\beta_{h d} S_{h}^{n+1} I_{d}^{n}-\left(\mu_{h}+\nu_{1}\right) S_{h}^{n+1} \\
\frac{E_{h}^{n+1}-E_{h}^{n}}{\phi(\Delta t)} & =\beta_{h d} S_{h}^{n+1} I_{d}^{n}-\left(\mu_{h}+\gamma_{h}+\nu_{2}\right) E_{h}^{n+1} \\
\frac{I_{h}^{n+1}-I_{h}^{n}}{\phi(\Delta t)} & =\gamma_{h} E_{h}^{n+1}-\left(\alpha_{h}+\mu_{h}\right) I_{h}^{n+1} \\
\frac{V_{h}^{n+1}-V_{h}^{n}}{\phi(\Delta t)} & =\nu_{1} S_{h}^{n+1}+\nu_{2} E_{h}^{n+1}-\mu_{h} V_{h}^{n+1} \\
\frac{S_{d}^{n+1}-S_{d}^{n}}{\phi(\Delta t)} & =\mu_{d} K_{d}-\beta_{d d} S_{d}^{n+1} I_{d}^{n}-\mu_{d} S_{d}^{n+1} \\
\frac{E_{d}^{n+1}-E_{d}^{n}}{\phi(\Delta t)} & =\beta_{d d} S_{d}^{n+1} I_{d}^{n}-\left(\mu_{d}+\gamma_{d}\right) E_{d}^{n+1} \\
\frac{I_{d}^{n+1}-I_{d}^{n}}{\phi(\Delta t)} & =\gamma_{d} E_{d}^{n+1}-\left(\alpha_{d}+\mu_{d}\right) I_{d}^{n+1}
\end{aligned}
$$

where the function $\phi(\Delta t)$ is obtained from the exact solutions of the conservation laws (2.8) and (2.9) to satisfies the condition

$$
\phi \equiv \phi(\Delta t)=\Delta t+\mathcal{O}\left((\Delta t)^{2}\right)
$$

In the approximation, positive constants are left as positive constants. From Equations (2.10) and (2.11), by using Grownwall inequality for difference equations, we choose

$$
\phi(\Delta t)=\frac{e^{\mu \Delta t}-1}{\mu} \quad \text { or } \quad \phi(\Delta t)=\frac{e^{(\mu+\alpha) \Delta t}-1}{\mu+\alpha},
$$

respectively, where $\mu=\max \left\{\mu_{h}, \mu_{d}\right\}$ and $\alpha=\max \left\{\alpha_{h}, \alpha_{d}\right\}$ (see [15, 19]). These functions satisfy Equation (4.8).

Remark 4.1 Note that in the constructed NSFD scheme, two Mickens rules [15] are reinforced as highlighted in [2]: the nonlinear terms are approximated in a nonlocal way and the usual standard denominator $\Delta t$ of the discrete derivative is replaced by the complex denominator function $\phi(\Delta t)$. 
By adding $(4.1)-(4.4)$ and $(4.5)-(4.7)$, we obtain

$$
\frac{N_{h}^{n+1}-N_{h}^{n}}{\phi(\Delta t)}=\mu_{h} K_{h}-\mu_{h} N_{h}^{n+1}-\alpha_{h} I_{h}^{n+1}
$$

and

$$
\frac{N_{d}^{n+1}-N_{d}^{n}}{\phi(\Delta t)}=\mu_{d} K_{d}-\mu_{d} N_{d}^{n+1}-\alpha_{d} I_{d}^{n+1}
$$

respectively. From (4.9) and (4.10), the inequalities

$$
\mu_{h} K_{h}-\left(\mu_{h}+\alpha_{h}\right) N_{h}^{n+1} \leq \frac{N_{h}^{n+1}-N_{h}^{n}}{\phi(\Delta t)} \leq \mu_{h} K_{h}-\mu_{h} N_{h}^{n+1}
$$

and

$$
\mu_{d} K_{d}-\left(\mu_{d}+\alpha_{h}\right) N_{d}^{n+1} \leq \frac{N_{d}^{n+1}-N_{d}^{n}}{\phi(\Delta t)} \leq \mu_{d} K_{d}-\mu_{d} N_{d}^{n+1}
$$

are obtained, respectively. By using the Gronwall inequality for discrete dynamical systems [18], solutions for (4.9) and (4.10) are positive and bounded. More precisely, $0 \leq N_{h}^{0} \leq K_{h}$ and $0 \leq N_{d}^{0} \leq K_{d}$, lead into the inequalities $0 \leq N_{h}^{n} \leq K_{h}$ and $0 \leq N_{d}^{n} \leq K_{d}$, respectively for $n \in \mathbb{N}$.

Theorem 4.2 The NSFD scheme (4.1) - (4.7) defines a discrete dynamical system on the biologically feasible domain

$$
\begin{aligned}
\Omega= & \left\{\left(S_{h}, E_{h}, I_{h}, V_{h}, S_{d}, E_{d}, I_{d}\right) \in \mathbb{R}^{7}: S_{h}, E_{h}, I_{h}, V_{h}, S_{d}, E_{d}, I_{d} \geq 0\right. \\
& \left.N_{h}, N_{d}>0,0 \leq S_{h}+E_{h}+I_{h}+V_{h}=N_{h} \leq K_{h}, 0 \leq S_{d}+E_{d}+I_{d}=N_{d} \leq K_{d}\right\} .
\end{aligned}
$$


For further computational purpose, we rearrange the system $(4.1)-(4.7)$ into

$$
\begin{aligned}
S_{h}^{n+1} & =\frac{\mu_{h} \phi(\Delta t) K_{h}+S_{h}^{n}}{1+\left(\beta_{h d} I_{d}^{n}+\mu_{h}+\nu_{1}\right) \phi(\Delta t)} \\
E_{h}^{n+1} & =\frac{\beta_{h d} \phi(\Delta t) S_{h}^{n+1} I_{d}^{n}+E_{h}^{n}}{1+\left(\mu_{h}+\gamma_{h}+\nu_{2}\right) \phi(\Delta t)} \\
I_{h}^{n+1} & =\frac{\gamma_{h} \phi(\Delta t) E_{h}^{n+1}+I_{h}^{n}}{1+\left(\alpha_{h}+\mu_{h}\right) \phi(\Delta t)} \\
V_{h}^{n+1} & =\frac{\left(\nu_{1} S_{h}^{n+1}+\nu_{2} E_{h}^{n+1}\right) \phi(\Delta t)+V_{h}^{n}}{1+\mu_{h} \phi(\Delta t)} \\
S_{d}^{n+1} & =\frac{\mu_{d} \phi(\Delta t) K_{d}+S_{d}^{n}}{1+\left(\beta_{d d} I_{d}^{n}+\mu_{d}\right) \phi(\Delta t)} \\
E_{d}^{n+1} & =\frac{\beta_{d d} \phi(\Delta t) S_{d}^{n+1} I_{d}^{n}+E_{d}^{n}}{1+\left(\mu_{d}+\gamma_{d}\right) \phi(\Delta t)} \\
I_{d}^{n+1} & =\frac{\gamma_{d} \phi(\Delta t) E_{d}^{n+1}+I_{d}^{n}}{1+\left(\alpha_{d}+\mu_{d}\right) \phi(\Delta t)}
\end{aligned}
$$

From Equations (4.13) - (4.19), we can see that the NSFD scheme (4.1) - (4.7) gives positive solutions for any positive initial data. Moreover, the algorithm runs by following the GaussSeidel cycle on $(4.13)-(4.19)$.

To determine the fixed points, we set $(4.13)$ - (4.19) into the following form:

$$
\begin{aligned}
\frac{\mu_{h} \phi(\Delta t) K_{h}+S_{h}}{1+\left(\beta_{h d} I_{d}+\mu_{h}+\nu_{1}\right) \phi(\Delta t)} & =S_{h} \\
\frac{\beta_{h d} \phi(\Delta t) S_{h} I_{d}+E_{h}}{1+\left(\mu_{h}+\gamma_{h}+\nu_{2}\right) \phi(\Delta t)} & =E_{h} \\
\frac{\gamma_{h} \phi(\Delta t) E_{h}+I_{h}}{1+\left(\alpha_{h}+\mu_{h}\right) \phi(\Delta t)} & =I_{h} \\
\frac{\left(\nu_{1} S_{h}+\nu_{2} E_{h}\right) \phi(\Delta t)+V_{h}}{1+\mu_{h} \phi(\Delta t)} & =V_{h} \\
\frac{\mu_{d} \phi(\Delta t) K_{d}+S_{d}}{1+\left(\beta_{d d} I_{d}+\mu_{d}\right) \phi(\Delta t)} & =S_{d} \\
\frac{\beta_{d d} \phi(\Delta t) S_{d} I_{d}+E_{d}}{1+\left(\mu_{d}+\gamma_{d}\right) \phi(\Delta t)} & =E_{d} \\
\frac{\gamma_{d} \phi(\Delta t) E_{d}+I_{d}}{1+\left(\alpha_{d}+\mu_{d}\right) \phi(\Delta t)} & =I_{d} .
\end{aligned}
$$


From (4.26), we get

$$
E_{d}=\frac{\left(\alpha_{d}+\mu_{d}\right) I_{d}}{\gamma_{d}}
$$

Plugging (4.27) into (4.25), we obtain

$$
\beta_{d d} S_{d} I_{d}=\frac{\left(\alpha_{d}+\mu_{d}\right)\left(\mu_{d}+\gamma_{d}\right) I_{d}}{\gamma_{d}} .
$$

From (4.28), we get

$$
I_{d}=0 \text { or } \quad S_{d}=\frac{\left(\alpha_{d}+\mu_{d}\right)\left(\mu_{d}+\gamma_{d}\right)}{\gamma_{d} \beta_{d d}}=\frac{K_{d}}{\mathcal{R}_{0}^{2}}
$$

If $I_{d}=0$, then

$$
E_{0}=\left(\frac{\mu_{h} K_{h}}{\mu_{h}+\nu_{1}}, 0,0, \frac{\nu_{1} K_{h}}{\mu_{h}+\nu_{1}}, K_{d}, 0,0\right)
$$

is the fixed point associated with the disease-free equilibrium point. This confirms that the scheme preserves the disease-free equilibrium point.

If $I_{d}>0$, by using $(3.10)$ and $(4.20)-(4.28), E_{\infty}=\left(S_{h}^{*}, E_{h}^{*}, I_{h}^{*}, V_{h}^{*}, S_{d}^{*}, E_{d}^{*}, I_{d}^{*}\right)$ is the endemic fixed point for $\mathcal{R}_{0}>1$, where

$$
\begin{aligned}
S_{d}^{*} & =\frac{K_{d}}{\mathcal{R}_{0}}, \\
I_{d}^{*} & =\frac{\mu_{d}\left(\mathcal{R}_{0}-1\right)}{\beta_{d d}}, \\
E_{d}^{*} & =\frac{\mu_{d}\left(\alpha_{d}+\mu_{d}\right)\left(\mathcal{R}_{0}-1\right)}{\gamma_{d} \beta_{d d}}, \\
S_{h}^{*} & =\frac{\mu_{h} \beta_{d d} K_{h}}{\mu_{d} \beta_{h d}\left(\mathcal{R}_{0}-1\right)+\left(\mu_{h}+\nu_{1}\right) \beta_{d d}}, \\
E_{h}^{*} & =\frac{\mu_{d} \mu_{h} \beta_{h d} K_{h}\left(\mathcal{R}_{0}-1\right)}{\left(\mu_{h}+\gamma_{h}+\nu_{2}\right)\left(\mu_{d} \beta_{h d}\left(\mathcal{R}_{0}-1\right)+\left(\mu_{h}+\nu_{1}\right) \beta_{d d}\right)}, \\
I_{h}^{*} & =\frac{\mu_{d} \mu_{h} \gamma_{h} \beta_{h d} K_{h}\left(\mathcal{R}_{0}-1\right)}{\left(\alpha_{h}+\mu_{h}\right)\left(\mu_{h}+\gamma_{h}+\nu_{2}\right)\left(\mu_{d} \beta_{h d}\left(\mathcal{R}_{0}-1\right)+\left(\mu_{h}+\nu_{1}\right) \beta_{d d}\right)}, \\
V_{h}^{*} & =\frac{K_{h}}{\mu_{d} \beta_{h d}\left(\mathcal{R}_{0}-1\right)+\left(\mu_{h}+\nu_{1}\right) \beta_{d d}}\left(\nu_{1} \beta_{d d}+\frac{\nu_{2} \mu_{d} \beta_{h d}\left(\mathcal{R}_{0}-1\right)}{\mu_{h}+\gamma_{h}+\nu_{2}}\right) .
\end{aligned}
$$

Hence, the NSFD scheme given in (4.1) - (4.7) also preserves the endemic equilibrium point. In the coming two theorems, we will check the stability properties of the fixed points. 
Theorem 4.3 The disease-free fixed point $E_{0}$ of $(4.1)-(4.7)$ is locally asymptotically stable if $\mathcal{R}_{0}<1$ and unstable if $\mathcal{R}_{0}>1$.

Proof: The Jacobian matrix of $(4.13)-(4.19)$ at $E_{0}$ is given by

$\left.\begin{array}{ccccccc}\frac{1}{1+\left(\mu_{h}+\nu_{1}\right) \phi} & 0 & 0 & 0 & 0 & 0 & x \\ 0 & \frac{1}{1+\left(\mu_{h}+\gamma_{h}+\nu_{2}\right) \phi} & 0 & 0 & 0 & 0 & y \\ 0 & z & \frac{1}{1+\left(\alpha_{h}+\mu_{h}\right) \phi} & 0 & 0 & 0 & 0 \\ \frac{\nu_{1}}{1+\mu_{h} \phi} \frac{\phi}{1+\left(\mu_{h}+\nu_{1}\right) \phi} & \frac{\nu_{2}}{1+\mu_{h} \phi} \frac{\phi}{1+\left(\mu_{h}+\gamma_{h}+\nu_{2}\right) \phi} & 0 & \frac{1}{1+\mu_{h} \phi} & 0 & 0 & -\frac{\beta_{d d} K_{d} \phi}{1+\mu_{d} \phi} \\ 0 & 0 & 0 & 0 & \frac{1}{1+\mu_{d} \phi} & 0 & \frac{\beta_{d d} K_{d} \phi}{1+\left(\mu_{d}+\gamma_{d}\right) \phi} \\ 0 & 0 & 0 & 0 & 0 & \frac{1}{1+\left(\mu_{d}+\gamma_{d}\right) \phi} & \frac{1}{1+\left(\mu_{d}+\gamma_{d}\right) \phi}\end{array}\right)$,

where

$$
\begin{aligned}
x & =\frac{-\mu_{h} \beta_{h d} K_{h} \phi}{\left(1+\left(\mu_{h}+\nu_{1}\right) \phi\right)\left(\mu_{h}+\nu_{1}\right)} \\
y & =\frac{\mu_{h} \beta_{h d} K_{h} \phi}{\left(1+\left(\mu_{h}+\gamma_{h}+\nu_{2}\right) \phi\right)\left(\mu_{h}+\nu_{1}\right)} \\
z & =\frac{\gamma_{h} \phi}{1+\left(\alpha_{h}+\mu_{h}\right) \phi} \frac{1}{1+\left(\mu_{h}+\gamma_{h}+\nu_{2}\right) \phi}
\end{aligned}
$$

The corresponding characteristic equation is

$$
\operatorname{det}\left(r I-J\left(E_{0}\right)\right)=0
$$


This leads into

$$
\begin{aligned}
\left(r-\frac{1}{1+\left(\mu_{h}+\nu_{1}\right) \phi}\right) & \left(r-\frac{1}{1+\left(\mu_{h}+\gamma_{h}+\nu_{2}\right) \phi}\right)\left(r-\frac{1}{1+\left(\alpha_{h}+\mu_{h}\right) \phi}\right) \\
& \left(r-\frac{1}{1+\mu_{h} \phi}\right)\left(r-\frac{1}{1+\mu_{d} \phi}\right)\left(r^{2}+a_{1} r+a_{0}\right)=0
\end{aligned}
$$

where

$$
\begin{aligned}
& a_{1}=-\left(\frac{1}{1+\left(\mu_{d}+\gamma_{d}\right) \phi}+\frac{1}{1+\left(\alpha_{d}+\mu_{d}\right) \phi}\right) \\
& a_{0}=\frac{1}{\left(1+\left(\mu_{d}+\gamma_{d}\right) \phi\right)\left(1+\left(\alpha_{d}+\mu_{d}\right) \phi\right)}\left(1-\frac{\mathcal{R}_{0}^{2}\left(\mu_{d}+\gamma_{d}\right)\left(\alpha_{d}+\mu_{d}\right) \phi^{2}}{1+\left(\mu_{d}+\gamma_{d}\right) \phi}\right)
\end{aligned}
$$

The stability properties of $E_{0}$ depends on the roots of

$$
r^{2}+a_{1} r+a_{0}=0
$$

By using [13], the roots lie inside the unit circle if and only if $\left|a_{0}\right|<1$ and $\left|a_{1}\right|<1+a_{0}$. Clearly $\left|a_{0}\right|<1$ and $\left|a_{1}\right|<1+a_{0}$ if $\mathcal{R}_{0}<1$. Hence, $E_{0}$ is locally asymptotically stable if $\mathcal{R}_{0}<1$ and unstable if $\mathcal{R}_{0}>1$.

Theorem 4.4 The disease-free fixed point $E_{0}$ is globally attractor if $\mathcal{R}_{0}<1$ and $\frac{\mu_{d}+\gamma_{d}}{\beta_{d d}} \leq 1$.

Proof: From Equation (4.18), we have

$$
\begin{aligned}
I_{d}^{n+1} & =\frac{\gamma_{d} \phi E_{d}^{n+1}+I_{d}^{n}}{1+\left(\alpha_{d}+\mu_{d}\right) \phi} \\
& \leq \frac{\left(\gamma_{d} \phi K_{d}+1\right) I_{d}^{n}}{1+\left(\alpha_{d}+\mu_{d}\right) \phi} \\
& =\left(\frac{\frac{\left(\alpha_{d}+\mu_{d}\right)\left(\mu_{d}+\gamma_{d}\right) \mathcal{R}_{0} \phi}{\beta_{d d}}+1}{1+\left(\alpha_{d}+\mu_{d}\right) \phi}\right) I_{d}^{n} .
\end{aligned}
$$

Thus, $I_{d}^{n+1} \leq D I_{d}^{n}$, where

$$
D=\frac{\frac{\left(\alpha_{d}+\mu_{d}\right)\left(\mu_{d}+\gamma_{d}\right) \mathcal{R}_{0}^{2} \phi}{\beta_{d d}}+1}{1+\left(\alpha_{d}+\mu_{d}\right) \phi} .
$$


For $\mathcal{R}_{0}<1$ and $\frac{\mu_{d}+\gamma_{d}}{\beta_{d d}} \leq 1, \quad 0<D<1$, the sequence $\left(I_{d}^{n}\right)_{n \geq 0}$ tends to zero for any initial value $0 \leq I_{d}^{0} \leq K_{d}$. Using this sequence in Equations (4.12) - (4.17), we can show that if $E_{n}=\left(S_{h}^{n}, E_{h}^{n}, I_{h}^{n}, V_{h}^{n}, S_{d}^{n}, E_{d}^{n}, I_{d}^{n}\right)$,

$$
\lim _{n \rightarrow \infty} E_{n}=E_{0}
$$

This implies that $E_{0}$ is globally attractor.

Theorem 4.5 The disease-free fixed point $E_{0}$ is globally asymptotically stable for $\mathcal{R}_{0}<1$ and $\frac{\mu_{d}+\gamma_{d}}{\beta_{d d}} \leq 1$.

Proof: This is a result obtained from Theorem 4.2 and Theorem 4.3.

Theorem 4.6 The endemic fixed point $E_{\infty}$ of $(4.1)-(4.7)$ is locally asymptotically stable for $\mathcal{R}_{0}>1$.

Proof: To prove this theorem, we need to show that all roots of the characteristic polynomial related to the Jacobian matrix of $(4.12)-(4.18)$ at $E_{\infty}$ lie inside the unit circle. To show this, we have the Jacobian matrix at $E_{\infty}$ and read as

$\left(\begin{array}{ccccccc}b & 0 & 0 & 0 & 0 & 0 & c \\ d & \frac{1}{1+\left(\mu_{h}+\gamma_{h}+\nu_{2}\right) \phi} & 0 & 0 & 0 & 0 \\ 0 & f & \frac{1}{1+\left(\alpha_{h}+\mu_{h}\right) \phi} & 0 & 0 & 0 \\ g & h & 0 & \frac{1}{1+\mu_{h} \phi} & 0 & 0 & 0 \\ 0 & 0 & 0 & 0 & \frac{1}{1+\left(\beta_{d d} I_{d}^{*}+\mu_{d}\right) \phi} & 0 & -\frac{\left(\mu_{d} \phi K_{d}+S_{d}^{*}\right) \beta_{d d} \phi}{\left(1+\left(\beta_{d d} I_{d}^{*}+\mu_{d}\right) \phi\right)^{2}} \\ 0 & 0 & 0 & & \frac{\beta_{d d} \phi I_{d}^{*}}{1+\left(\mu_{d}+\gamma_{d}\right) \phi} \frac{1}{1+\left(\beta_{d d} I_{d}^{*}+\mu_{d}\right) \phi} & \frac{1}{1+\left(\mu_{d}+\gamma_{d}\right) \phi} & \frac{\beta_{d d} \phi S_{d}^{*}}{1+\left(\mu_{d}+\gamma_{d}\right) \phi} \\ 0 & 0 & 0 & & 0 & \frac{\gamma_{d} \phi}{1+\left(\alpha_{d}+\mu_{d}\right) \phi} \frac{1}{1+\left(\mu_{d}+\gamma_{d}\right) \phi} & \frac{1}{1+\left(\alpha_{d}+\mu_{d}\right) \phi}\end{array}\right)$


where,

$$
\begin{aligned}
b & =\frac{1}{1+\left(\beta_{h d} I_{d}^{*}+\mu_{h}+\nu_{1}\right) \phi} \\
c & =-\frac{\left(\mu_{h} \phi K_{h}+S_{h}^{*}\right) \beta_{h d} \phi}{\left(1+\left(\beta_{h d} I_{d}^{*}+\mu_{h}+\nu_{1}\right) \phi\right)^{2}} \\
d & =\frac{\beta_{h d} \phi I_{d}^{*}}{1+\left(\mu_{h}+\gamma_{h}+\nu_{2}\right) \phi} \frac{1}{1+\left(\beta_{h d} I_{d}^{*}+\mu_{h}+\nu_{1}\right) \phi} \\
e & =\frac{\beta_{h d} \phi S_{h}^{*}}{1+\left(\mu_{h}+\gamma_{h}+\nu_{2}\right) \phi} \\
f & =\frac{\mu_{h} \phi}{1+\left(\alpha_{h}+\mu_{h}\right) \phi} \frac{1}{1+\left(\mu_{h}+\gamma_{h}+\nu_{2}\right) \phi} \\
g & =\frac{\nu_{1} \phi}{1+\mu_{h} \phi} \frac{1}{1+\left(\beta_{h d} I_{d}^{*}+\mu_{h}+\nu_{1}\right) \phi} \\
h & =\frac{\nu_{2} \phi}{1+\mu_{h} \phi} \frac{1}{1+\left(\mu_{h}+\gamma_{h}+\nu_{2}\right) \phi} .
\end{aligned}
$$

For an eigenvalue $r$ of $E_{\infty}$, the characteristic equation is given by

$$
\operatorname{det}\left(r I-J\left(E_{\infty}\right)\right)=0
$$

By observing the given Jacobian matrix, the stability properties of $E_{\infty}$ are determined based on the roots of the cubic equation

$$
\begin{array}{r}
\left(r-\frac{1}{1+\left(\beta_{d d} I_{d}^{*}+\mu_{d}\right) \phi}\right)\left\{\left(r-\frac{1}{1+\left(\mu_{d}+\gamma_{d}\right) \phi}\right)\left(r-\frac{1}{1+\left(\alpha_{d}+\mu_{d}\right) \phi}\right)-\frac{\gamma_{d} \beta_{d d} \phi^{2} S_{d}^{*}}{\left(1+\left(\mu_{d}+\gamma_{d}\right) \phi\right)^{2}\left(1+\left(\alpha_{d}+\mu_{d}\right) \phi\right)}\right\} \\
+\frac{\left(\mu_{d} \phi K_{d}+S_{d}^{*}\right) \gamma_{d} \beta_{d d}^{2} \phi^{3} I_{d}^{*}}{\left(1+\left(\beta_{d d} I_{d}^{*}+\mu_{d}\right) \phi\right)^{3}\left(1+\left(\mu_{d}+\gamma_{d}\right) \phi\right)^{2}\left(1+\left(\alpha_{d}+\mu_{d}\right) \phi\right)}=0,
\end{array}
$$

the other roots of the characteristic equation lie inside the unit circle. This expression can be simplified into

$$
r^{3}+a_{2} r^{2}+a_{1} r+a_{0}=0
$$


where,

$$
\begin{aligned}
a_{2} & =-\left(\frac{1}{1+\left(\mu_{d}+\gamma_{d}\right) \phi}+\frac{1}{1+\left(\alpha_{d}+\gamma_{d}\right) \phi}+\frac{1}{1+\left(\beta_{d d} I_{d}^{*}+\mu_{d}\right) \phi}\right) \\
a_{1} & =\left(\frac{1}{1+\left(\beta_{d d} I_{d}^{*}+\mu_{d}\right) \phi}\right)\left(\frac{1}{1+\left(\mu_{d}+\gamma_{d}\right) \phi}+\frac{1}{1+\left(\alpha_{d}+\gamma_{d}\right) \phi}\right) \\
& +\frac{1}{\left(1+\left(\mu_{d}+\gamma_{d}\right) \phi\right)\left(1+\left(\alpha_{d}+\gamma_{d}\right) \phi\right)}\left(1-\frac{\frac{\gamma_{d} \beta_{d d} K_{d}}{\mathcal{R}_{0}} \phi^{2}}{1+\left(\mu_{d}+\gamma_{d}\right) \phi}\right) \\
a_{0} & =\frac{1}{\left(1+\left(\beta_{d d} I_{d}^{*}+\mu_{d}\right) \phi\right)} \frac{1}{\left(1+\left(\mu_{d}+\gamma_{d}\right) \phi\right)\left(1+\left(\alpha_{d}+\gamma_{d}\right) \phi\right)}\left(\frac{\frac{\gamma_{d} \beta_{d d} K_{d}}{\mathcal{R}_{0}} \phi^{2}}{1+\left(\mu_{d}+\gamma_{d}\right) \phi}-1\right)+c
\end{aligned}
$$

where,

$$
c=\frac{\left(\mu_{d} \phi K_{d}+S_{d}^{*}\right) \gamma_{d} \beta_{d d}^{2} I_{d}^{*} \phi^{2}}{\left(1+\left(\beta_{d d} I_{d}^{*}+\mu_{d}\right) \phi\right)^{3}\left(1+\left(\alpha_{d}+\mu_{d}\right) \phi\right)\left(1+\left(\mu_{d}+\gamma_{d}\right) \phi\right)^{2}} .
$$

By using [13], $E_{\infty}$ is locally asymptotically stable if the the roots of (4.30) lie inside the unit circle. This is true if and only if

$$
\left|a_{0}+a_{2}\right|<1+a_{1} \text { and } \quad\left|a_{1}-a_{0} a_{2}\right|<1-a_{0}^{2} .
$$

From the given values, we can check that $\left|a_{0}+a_{2}\right|<1+a_{1}$ and $\left|a_{1}-a_{0} a_{2}\right|<1-a_{0}^{2}$ for $\mathcal{R}_{0}>1$. Hence, $E_{\infty}$ is locally asymptotically stable for $\mathcal{R}_{0}>1$.

\section{Numerical Simulation}

In this section, we give numerical experiments for our nonstandard finite difference scheme (4.1)-(4.7). It is known that standard finite difference methods do not always preserve the dynamics of the corresponding differential equation (see [2]). The designed numerical scheme, for arbitrary values of the system parameters, provides accurate numerical solutions to the original model. To show this, we arbitrarily choose the data given in Table 2 with in the domain of parameters to show the consistency of the scheme with the theoretical analysis of the continuous model. By using Eqn (3.10), the corresponding value of $\mathcal{R}_{0} \simeq 3.3>1$. Hence, by using Theorem 4.5, we can infer that all solutions will converge to the endemic fixed point 
$E_{\infty}$. More precisely, the performance of the NSFD scheme (4.1) - (4.7) is illustrated in Fig 2-3, which display the local stability of endemic fixed point for $\mathcal{R}_{0}>1$. For $\mathcal{R}_{0}<1$, analogously the disease-free fixed point is globally asymptotically stable.

\begin{tabular}{|c|c|c|c|}
\hline Variable & value & parameter & value \\
\hline$K_{h}$ & 1000 & $\beta_{h d}$ & 0.3 \\
\hline$S_{h} 0$ & 900 & $\mu_{h}$ & 0.2 \\
\hline$E_{h} 0$ & 0 & $\alpha_{h}$ & 0.1 \\
\hline$I_{h} 0$ & 0 & $\gamma_{h}$ & 0.2 \\
\hline$V_{h} 0$ & 10 & $\nu_{1}$ & 0.2 \\
\hline$K_{d}$ & 100 & $\nu_{2}$ & 0.1 \\
\hline$S_{d} 0$ & 80 & $\beta_{d d}$ & 0.5 \\
\hline$E_{d} 0$ & 5 & $\mu_{d}$ & 0.1 \\
\hline$I_{d} 0$ & 5 & $\gamma_{d}$ & 0.2 \\
\hline & & $\alpha_{d}$ & 0.2 \\
\hline
\end{tabular}

Table 2: parameter values.

\section{Conclusion}

The qualitative analysis of the rabies model is given. It is proved that the disease free equilibrium is globally stable for $\mathcal{R}_{0}<1$ and unstable for $\mathcal{R}_{0}>1$. The endemic equilibrium is locally asymptotically stable for $\mathcal{R}_{0}>1$.

Nonstandard finite difference techniques were developed empirically for solving practical problems in applied sciences before two decades by R. E. Mickens. Having this in our mind, we designed a NSFD scheme which is dynamically consistent with the original rabies model. Numerical simulations of the NSFD scheme are given from Fig 2-3.

The given model is relatively simple and it captures some basic features of epidemiological 


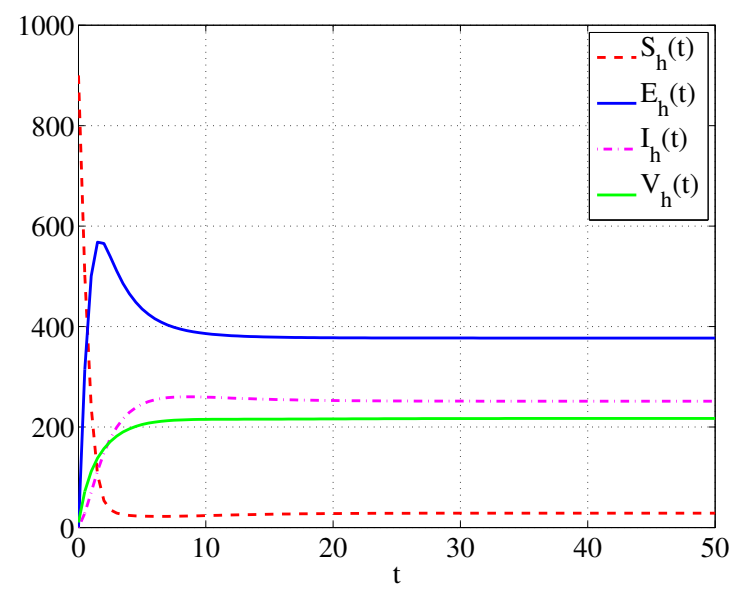

Figure 2: Numerical simulation of human population

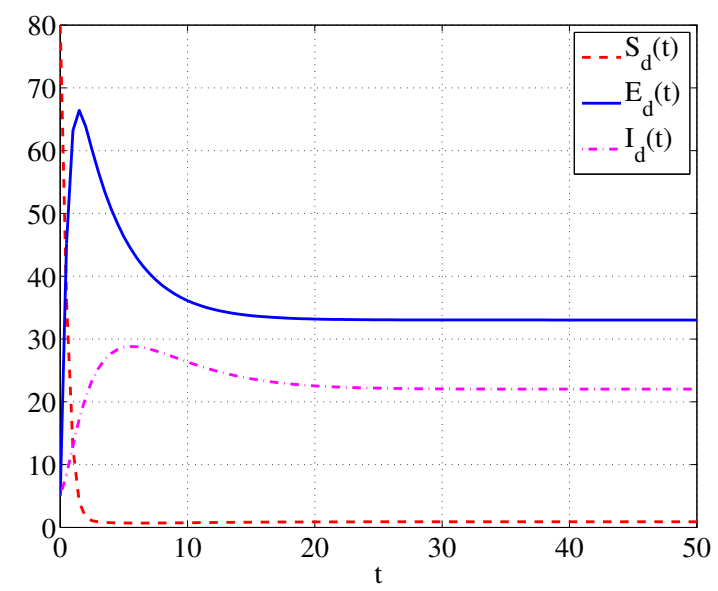

Figure 3: Numerical simulation of dog population

models. When the rabies virus enters the central nervous system, it induces behavioral changes in its host. If it enters in the spinal cord, it causes paralysis. Moreover, if it enters the limbic system, the dog become aggressive, lose their sense of direction and territorial behavior and wander about in a more or less random way. For such cases, our future interest is to add 
a diffusion term in the infected dog compartment and to add vaccination parameters in the susceptible dog population. Fitting real data to estimate the parameters is also our interest for future research. The extension of the model will also include the qualitative analysis and the numerical simulation.

Acknowledgments: The authors acknowledge the financial support of the DST/NRF SARChI Chair in Mathematics Models and Methods in Bioengineering and Biosciences. Thanks are also addressed to the two anonymous referees and to the editor-in- chief of the journal for their comments and suggestions.

\section{References}

[1] R. Anguelov, Y. Dumont, J. M. - S. Lubuma and E. Mureithi, Stability analysis and dynamics preserving NSFD schemes for a malaria model, Mathematical Population Studies, 20(2013), 101-122.

[2] R. Anguelov and J. M. - S. Lubuma, Contribution to the mathematics of the nonstandard finite difference method and application, Numerical Methods and Partial Differential Equations 17(5) (2001), 518-543.

[3] H. L. Bayer, Epidemiological models of rabies in domestic dogrs: dynamics and control, $\mathrm{PhD}$ thesis, University of Glasgow, 2010.

[4] F. Brauer, P. van den Driessche and Jianhong Wu (Eds.), Mathematical Epidemiology. Mathematical Biosciences Subseries, LNM 1945, Springer, 2008.

[5] S. Busenberg and K. Cooke, Verically Transmitted Disease: Models and Dynamics, Springer-Verlag, Vol. 23, 1993.

[6] O. Diekmann and J.A. Heesterbeek, Mathematical epidemiology of infectious diseases, Wiley, 2000. 
[7] M. Fekadu, Rabies in Ethiopia, American Journal of Epdemiology, 115 (2), 266-273.

[8] F. R. Gantmacher, The Theory of Matrices, Vol. 2, Chelsea (New York), 1959.

[9] K. Hampson, et al, Synchronous cycles of domestic dog rabies in Sub-Saharan Africa and the impact of control efferts, The National Academic of Sciences of the USA, 104 (18), 2007, 7717-7722.

[10] W. Haupt, Rabies-risk of exposure and current trends in preventation of human cases, Vaccine, 17 (1999), 1742-1749.

[11] Jackson et al, Management of Rabies in Humans, Clinical Infectious Diseases, 36 (2003), 60-63

[12] J. P. Lasalle, The Stability of Dynamical Systems, SIAMS, 1976.

[13] J. P. Lasalle, The Stability and Control of Discrete Discrete Processes, Springer-Verlag, 1986.

[14] J. M. - S., Lubuma, E. W. Mureithi and Y. A. Terefe, Analysis and dynamically consistent numerical schemes for the SIS model and related reaction diffusion equation. In CI Christov and MDTodorov (eds), Proceedings of the 3rd International Conference on Application of Mathematics in Technical and Natural Sciences (AMiTaNS11), (Albena, Bulgaria), American Institute of Physics - AIP Conference Proceedings 1404, 2011, 168175.

[15] R. E. Mickens, Nonstandard Finite Difference Methods of Differential Equations, World Scientific, Singapore, 1994.

[16] R. E. Mickens, Calculation of denominator functions for nonstandard finite difference schemes for differential equations satisfying a positive condition, Numerical Methods for Partial Differential Equations, 23 (2007), 672-691. 
[17] J. D. Murray, Mathematical Biology II: Spatial models and Biomedical Applications. Springer, Singapore, 2003.

[18] A. M. Stuart and A. M. Humphties, Dynamical systems and numerical analysis, Cambridge University Press, Cambridge, 1998.

[19] Y. A. Terefe, Bifurcation analysis and nonstandard finite difference schemes for Kermack and McKendrick type epidemiological models, MSc dissertation, University of Pretoria, Pretoria, 2012.

[20] P. van der Driessche and J. Watmough, Further notes on the basic reproduction number, in: F. Brauer, P. van der Driessche, J. Wu(eds), Mathematical epidemiology, Lecture Notes in Mathematical Biosciences Subseries, Vol. 1945, Springer, 2008, 159-178. 